\title{
Euphoriant effects of nicotine in smokers
}

\author{
Cynthia S. Pomerleau and Ovide F. Pomerleau \\ Behavioral Medicine Research Laboratory, Department of Psychiatry, University of Michigan, \\ Riverview Medical Building, 900 Wall Street, Ann Arbor, MI 48105, USA
}

Received July 18, 1991 / Final version April 30, 1992

\begin{abstract}
Two studies were conducted to replicate and extend previous demonstrations of smoking-induced, dose-related reports of euphoria, and to confirm this relationship using measures of plasma nicotine. In experiment 1 , overnight-deprived subjects, in three different sessions, smoked ultralow-, high-nicotine, and usualbrand cigarettes. In experiment 2, ultralow-, medium-, and high-nicotine cigarettes were used, and plasma nicotine was measured. In both studies, subjects were asked to depress a button during euphoric sensations. Number of sensations for the ultralow-nicotine cigarette was significantly lower than for the high-nicotine cigarette in the first study, and than for both the medium- and highnicotine conditions in the second; a significant linear trend was observed for number of sensations as a function of plasma nicotine level in the second study. For the high-nicotine cigarette, 19 of 22 subjects experienced at least one sensation (mean around three), starting around $2.5 \mathrm{~min}$ after lighting up. Together, these studies support the existence of a dose-response relationship for nicotine-induced euphoric sensations; suggest that they are more pronounced following overnight abstinence than following minimal deprivation, and in more dependent smokers; and characterize in detail the temporal features of these sensations.
\end{abstract}

Key words: Cigarette smoking - Dose-related - Euphoriant effects - Nicotine

Though early conceptualizations of " addiction" stressed the development of tolerance and the emergence of characteristic symptomatology upon withdrawal, most theorists now accept a broader definition of addiction that encompasses the pleasurable effects of a drug as well. The extent to which such sensations are associated with nicotine use, however, and to which they contribute to smoking behavior, are not well understood. The rela-

Correspondence to: C.S. Pomerleau tive acceptability of nicotine in the past, compared with drugs like cocaine or amphetamines, is to some degree attributable to its failure to produce "highs" of sufficient magnitude to disrupt ongoing activity. In a study of around 1000 people seeking treatment for drug dependence, Kozlowski et al. (1989) found that cigarettes were rated as less pleasurable than alcohol or other drugs. In animals, the range of conditions under which nicotine demonstrates reinforcing stimulus properties is much more restricted than those for psychomotor stimulant, opiate, and sedative-hypnotic drugs (Dougherty et al. 1981).

On the other hand, nicotine has repeatedly been shown to produce EEG changes suggestive of cortical activation in both animals and humans, whether delivered intravenously, via smoking, or via nicotine gum (see USDHHS 1988); the response is partially prevented by the nicotinic cholinergic antagonist mecamylamine (Pickworth et al. 1988), and tolerance develops rapidly (Hubbard and Gohd 1975). A series of studies carried out at the Addiction Research Center of the National Institute on Drug Abuse indicated that intravenous nicotine injections produced dose-related decreases in alpha EEG activity, with multiple short episodes of euphoria appearing within $30 \mathrm{~s}$ of the injection (Jasinski et al. 1984). Nicotine, whether administered via smoking or intravenously, has been shown to produce dose-related increases in scores on drug liking and euphoria, brief in duration, fairly rapid in onset (for smoking, occurring within a minute after completion of a cigarette), and showing the development of acute tolerance (Henningfield 1984; Henningfield et al. 1987): intravenous infusion produces a "rush" similar, though shorter in duration, to that produced by cocaine or morphine (Henningfield and Jasinski 1983; Henningfield et al. 1986). Liking scores are attenuated by mecamylamine in a dose-related manner (Henningfield 1983).

This paper reports the results of two studies designed 1) to replicate previous reports that smoking and nicotine administration produce dose-related euphoria by asking subjects to depress a button when they experi- 
enced euphoric sensations, and to confirm this relationship using direct measures of plasma nicotine; 2) to tease out the effects of nondrug factors by comparing a usualbrand cigarette with a research cigarette; 3 ) to examine the effects of abstinence versus minimal deprivation upon these sensations; 4) to assess the possible role of degree of dependence; and 5) to characterize the temporal features of such sensations. The apparatus used, a simplified version of a device used by Lukas and colleagues to investigate the euphoriant effects of intravenous nicotine, alcohol, and other drugs (Lukas et al. 1986), was intended to maximize our ability to capture evanescent effects by providing a continuous opportunity for subject-initiated reporting rather than periodic investigator-initiated querying. Experiment 1 was a preliminary study designed to verify the existence of the phenomenon under investigation and to determine which variables were likely to be of greatest interest. Subjects in this study smoked an ultralow-nicotine research cigarette (the control condition) in one session, a high-nicotine research cigarette in another session, and a usual-brand cigarette in a third (to assess the contribution of "familiarity" or other non-nicotine factors to the phenomenon, as well as to establish that it was part of the experiential repertoire of smokers under habitual smoking conditions). Both the first cigarette of the day and a second cigarette, half an hour later, were assessed. Based on the results of this study, a follow-up study was conducted in which blood samples were collected in order to determine actual nicotine intake and to attempt to relate nicotine levels to euphoria more systematically. Cigarettes of three distinct strengths (ultralow-, medium-, and high-nicotine) were used in an effort to establish a parametric dose-response relationship. Because the first cigarette of the day produced the most pronounced effects in the initial study, only that cigarette was tested in the second study.

\section{Materials and methods}

\section{Subjects}

Subjects for experiment 1 were ten male smokers recruited from the local community. Inclusion and exclusion criteria were as follows: age $20-40$ years; smoked at least 5 years; Fagerstrom Tolerance Questionnaire (FTQ, a measure of nicotine dependence with possible scores ranging from 0 to 11; Fagerstrom 1978) at least 5; usual brand of cigarettes nonmenthol and having a nicotine yield of $\geq 0.5 \mathrm{mg}$; no history of high blood pressure or cardiovascular problems; and not on any medications. Subjects for experiment 2 were 12 male smokers selected using the same inclusion and exclusion criteria except that minimum number of years smoked was 3 rather than 5 and nicotine yield of the subject's usual-brand cigarette was not restricted.

\section{Apparatus}

During experimental sessions, subjects were seated in an easy chair in a room equipped with a one-way mirror. Heart-rate was monitored and recorded using a Grass Polygraph (Model 7 B). All exper- imental sequences were controlled by an IBM AT computer. Standardized instructions were delivered via an Amiga computer equipped with a voice synthesizer; the Amiga was also used to present visual analogue scales, to which the subject responded using a mouse. No subject-experimenter intraction took place once a session began. During experiment 2, which involved blood sampling, an indwelling 18-gauge catheter was inserted in a left forearm vein and attached to a $1 \mathrm{~m}$ length of infusion-exfusion tubing that ran through a channel in the wall to allow unobtrusive withdrawal in the adjacent room; the line was heparinized, and samples were collected in tubes impregnated with EDTA to prevent clotting. They were kept on ice during the session, then centrifuged at $4^{\circ} \mathrm{C}$, and kept frozen at $-80^{\circ} \mathrm{C}$. Samples were assayed for nicotine and cotinine using HPLC (Hariharan et al. 1988).

Standardized unfiltered high-nicotine $(2.4 \mathrm{mg})$, medium-nicotine $(1.3 \mathrm{mg})$ and ultralow-nicotine research cigarettes $(0.2 \mathrm{mg})$ used in these studies were manufactured by the Tobacco and Health Research Institute, University of Kentucky (2R1, 1A3, and 3A1, respectively). (The ultralow-nicotine cigarette was intended as a control condition and expected to produce minimal nicotine intake; the rationale was to help maintain subject blindness and to avoid the possible "psychoactive" effects of non-nicotine substitutes.) Puff number and duration were measured using a gauge pressure transducer (LX 160-46; National Semiconductor); pressure changes produced by inhaling through a modified cigarette holder were transmitted to the pressure sensor via flexible plastic tubing, where they were converted to digital electric signals. Euphoric sensations were reported by the subject's pressing, for the duration of the sensation, a push-button with output to the AT, which computed time of onset and duration of each button press.

\section{Procedure}

Experiment 1. Subjects were screened by telephone. If they appeared to meet eligibility requirements and wished to participate in the study after hearing it described, they were scheduled for three 1 -h sessions separated by at least 1 day.

Subjects were requested to arrive at the lab at 8:00 a.m., without having smoked or ingested caffeine after midnight, and having eaten a standard breakfast (across the three sessions). Before the first session, the apparatus was demonstrated and a written informed consent document was obtained. Subjects were giving the following instructions for reporting euphoriant effects: "People sometimes report experiencing pleasurable sensations when they smoke that might be described as a rush, a buzz, or a high. Not everybody experiences these, and not all cigarettes produce these sensations. We are currently testing different blends of tobacco to determine how likely they are to produce these sensations. If you happen to experience any of these pleasurable sensations while smoking today, please depress the button and hold it down for the duration of the sensation." Electrodes were emplaced for collection of EKG data.

At the start of the session, the subject was asked to smoke a cigarette, using the cigarette holder designed to record topography, and to depress the button to report a buzz as described above. For the next half hour, the subject was permitted to read a magazine. The subject then smoked a second cigarette identical to the first one, following the same procedure as for the first cigarette.

Subjects were exposed to three sessions, separated by at least 1 day. The first two, counterbalanced to control for order effects, involved a high-nicotine research cigarette on one day and an ultralow-nicotine research cigarette on the other. During the third session - always the last, to avoid possible contamination of our assessment of strictly dose-related effects by interspersing a condition in which nondrug factors might influence the dependent measure - the subject smoked his usual-brand cigarettes. Sessions 1 and 2 were run double-blind; session 3 was not. Subjects were paid $S 45$ upon completion of the three sessions. 
Experiment 2. Subjects who appeared to meet inclusion criteria and expressed a wish to participate in the study were invited to a 15-min familiarization, during which they were introduced to the experimental apparatus and asked to sign an informed consent document. They were then scheduled for three experimental sessions.

Subjects were asked to report to the lab at $8: 30 \mathrm{a} . \mathrm{m}$., with instructions to abstain from smoking and caffeine intake after midnight and to eat a standard breakfast. For all sessions, expired CO was tested, and any subject with a reading $\geq 20$ ppm was dismissed. After insertion of an indwelling catheter and emplacement of electrodes for cardiac monitoring, subjects were permitted to read magazines during a $30 \mathrm{~min}$ acclimation period. The subject was then asked to smoke, using the device for measuring topography and reporting sensations of euphoria by pressing the button as described above.

Subjects were exposed to three sessions, scheduled 2-3 days apart. Sessions, presented in Latin square order to control for order effects, involved three cigarette conditions: ultralow-, medium-, and high-nicotine research cigarettes. Both subject and experimenter were blind to experimental condition throughout. Subjects were paid $\$ 50$ at the completion of the three sessions.

\section{Data analysis}

The principal data analyses wer carried out using repeated measures factorial ANOVAs; Tukey's HSD test was used as appropriate to specify differences among means. In addition, data from the two subject samples for the first cigarette of the day in the session when the high-nicotine cigarette was smoked, the only condition common to the two studies, were combined in an attempt 1) to specify further the number and temporal characteristics of euphoric sensations and their relationship to smoking topography, and 2) to determine whether individual differences in smoking patterns might predict likelihood of experiencing euphoric sensations in response to smoking. In addition to the number and total duration of button-presses, we calculated the average duration of a euphoric sensation, the mean number of puffs before the occurrence of the first such sensation, the interval of time that elapsed from the onset of the first puff to the onset of the first euphoric sensation, and the interval of time between onset of the first euphoric sensation and the onset of the puff immediately preceding that sensation. Number and total duration of euphoric sensations were correlated with age, years smoked, FTQ score, cotinine level (for the 12 subjects in experiment 2 only), and total puff number.

\section{Results}

Subjects in experiment 1 had a mean age $( \pm S D)$ of $27.7 \pm 6.5$ years, had smoked a mean of $11.0 \pm 5.7$ years, smoked an average of $24.5 \pm 6.0$ cigarettes/day with a mean nicotine yield of $0.94 \pm 0.25 \mathrm{mg}$, and had mean FTQ scores of $6.2 \pm 1.5$. Subjects in experiment 2 had a mean age of $29.4 \pm 6.4$ years, had smoked a mean of $11.9 \pm 8.1$ years, and smoked an average of $22.2 \pm$ 5.8 cigarettes/day with a mean nicotine yield of $0.85 \pm$ $0.31 \mathrm{mg}$. They had mean FTQ scores of $6.7 \pm 1.4$ and baseline cotinine levels of $245.7 \pm 148.8 \mathrm{ng} / \mathrm{ml}$. FTQ scores and, in experiment 2, cotinine levels characterize both subject samples as moderately dependent smokers. The two samples did not differ significantly on any parameters. All subjects in both studies readily understood the definition of a euphoric sensation provided, and none expressed any reservations about applying it to the experience of cigarette-smoking.

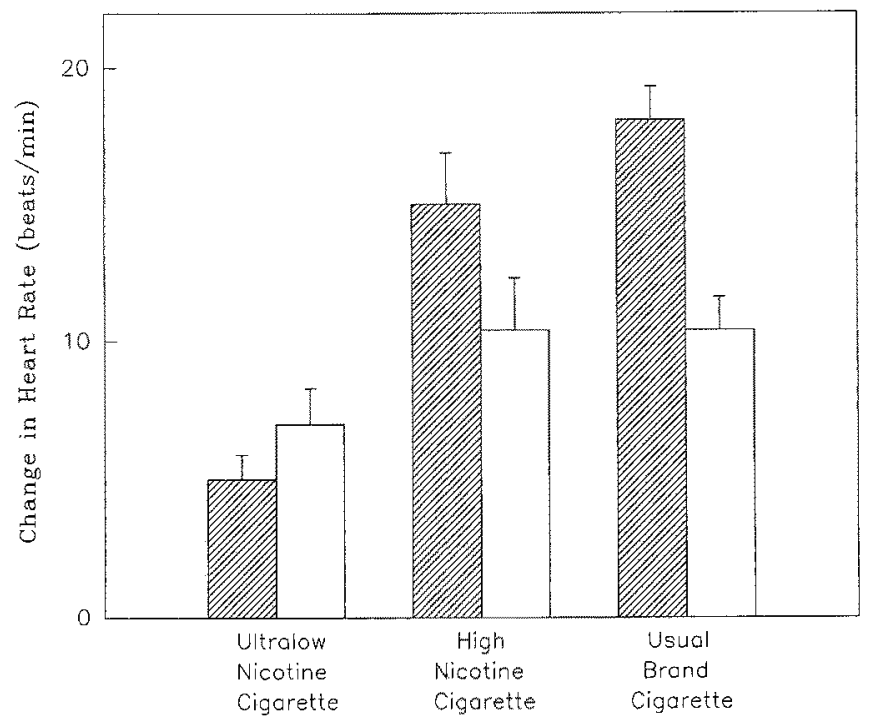

Fig. 1. Change in heart rate after smoking ultralow-nicotine research cigarettes, high-nicotine research cigarettes, and usual-brand cigarettes as the first $(\square)$ and second $(\square)$ cigarettes of the day (mean $\pm \mathrm{SEM} ; N=10$ )

\section{Experiment 1}

Although nicotine intake was not measured in this study, pre- to post-smoking heart rate boost (see Fig. 1) showed highly significant differences for cigarette condition [ultralow-nicotine, high-nicotine, and usual-brand cigarettes; $F(2,18)=33.04, P<0.0001$ ], ordinal position [first versus second cigarette; $F(1,9)=12.56, P<0.01]$, and condition $\times$ position $[F(2,18)=7.44, P<0.005]$. For the first cigarette of the day, heart-rate boosts for both the high-nicotine and usual-brand cigarettes were significantly greater than that for the low-nicotine cigarette (high versus low: $P<0.01$; usual versus low: $P<0.01$ ); the boost for the usual-brand cigarette did not differ significantly from that for the high-nicotine cigarette. For the second cigarette of the day, heart rate boosts did not differ significantly among the three cigarettes. For the usual-brand cigarette, the boost was significantly greater for the first cigarette of the day $(P<0.01)$.

In the ultralow-nicotine condition, only four subjects $(40 \%)$ reported experiencing euphoric sensations while smoking the first cigarette of the day and two $(20 \%)$ during the second. In the high-nicotine condition, eight subjects $(80 \%)$ reported euphoric sensations during the first cigarette, and four $(40 \%)$ during the second. Corresponding numbers for the usual-brand cigarette were six $(60 \%)$ and three $(30 \%)$.

Figure 2 (upper panel) shows mean number of reports of euphoric sensations for each condition. Significant differences were observed for ordinal position $[F(1,9)=$ $10.80, P<0.01]$, and for condition $\times$ position interaction $[F(2,18)=5.18, P<0.05]$. These effects are almost entirely accounted for by the elevation in number of reports of euphoric sensations for the first high-nicotine research cigarette of the day, which was significantly greater than for the first ultralow-nicotine cigarette of the day $(P<$ 0.05 ), as well as for the second high-nicotine cigarette 

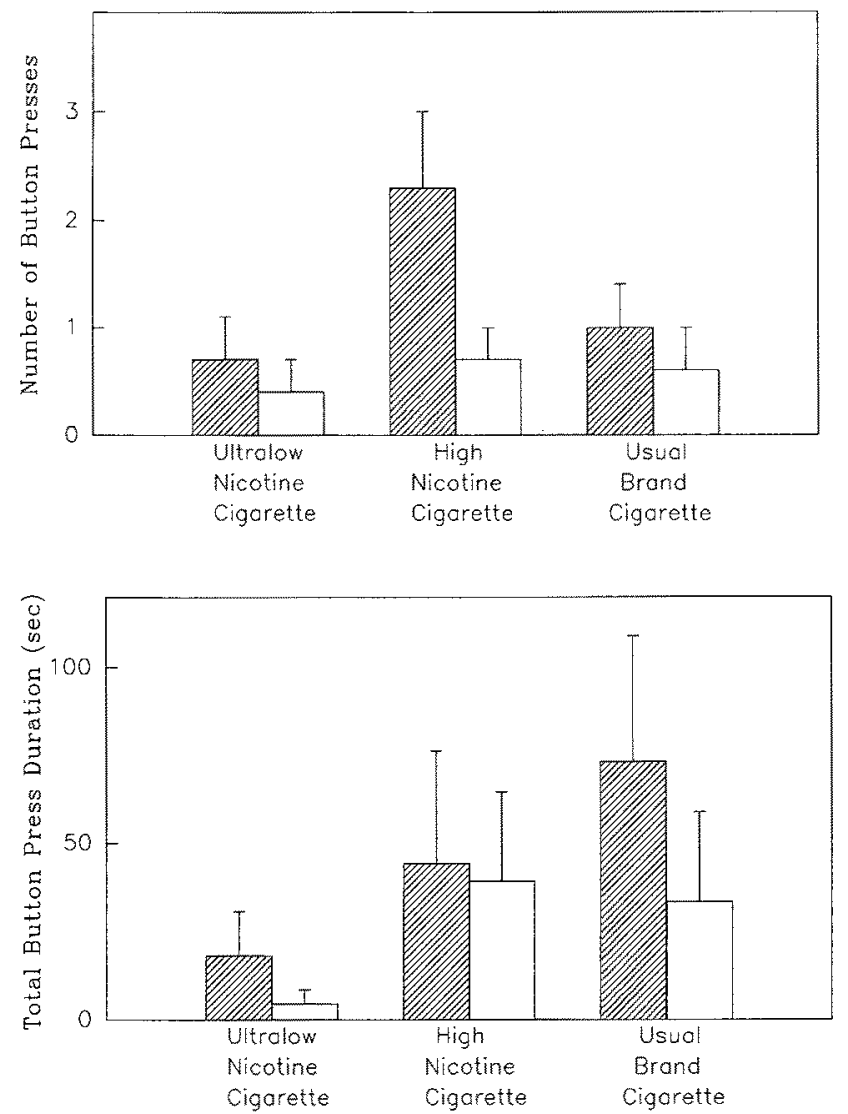

Fig. 2. Upper panel: number of reports of euphoric sensations (button-presses) associated with smoking ultralow-nicotine research cigarettes, high-nicotine research cigarettes, and usual-brand cigarettes as the first (@) and second ( $\square$ ) cigarettes of the day (mean \pm SEM; $N=10$ ). Lower panel: total duration of reports of euphoric sensations (time during which button was depressed) associated with smoking ultralow-nicotine research cigarettes, high-nicotine research cigarettes, and usual-brand cigarettes as the first ( $\square)$ and second ( $\square$ ) cigarettes of the day (mean \pm SEM $N=10$ )

$(P<0.05)$. Figure 2 (lower panel) shows total duration of reported euphoric sensations; no significant main or interaction effects were observed.

\section{Experiment 2}

Mean pre-smoking nicotine levels were $3.6 \pm 0.9 \mathrm{ng} / \mathrm{ml}$ (maximum $9 \mathrm{ng} / \mathrm{ml}$ ), $2.8 \pm 1.1 \mathrm{ng} / \mathrm{ml}$ (maximum $11 \mathrm{ng} /$ $\mathrm{ml}$ ) and $2.9 \pm 0.8 \mathrm{ng} / \mathrm{ml}$ (maximum $7 \mathrm{ng} / \mathrm{ml}$ ) for the ultralow-, medium-, and high-nicotine sessions, respectively, indicating good compliance with the instruction not to smoke after midnight on the previous night.

Though mean $\pm S E M$ plasma nicotine increment was low for the ultralow-nicotine condition $(4.5 \pm 1.0 \mathrm{ng} / \mathrm{ml})$, subjects foiled our attempt to administer parametric doses of nicotine by producing almost identical mean increments for the medium- and high-nicotine cigarettes $(18.7 \pm 3.5 \mathrm{ng} / \mathrm{ml}$ and $17.5 \pm 2.5 \mathrm{ng} / \mathrm{ml}$, respectively). A corresponding pattern was observed for both number and duration of button-presses, with means that were low for ultralow-nicotine cigarette $(1.3 \pm 1.0$ button
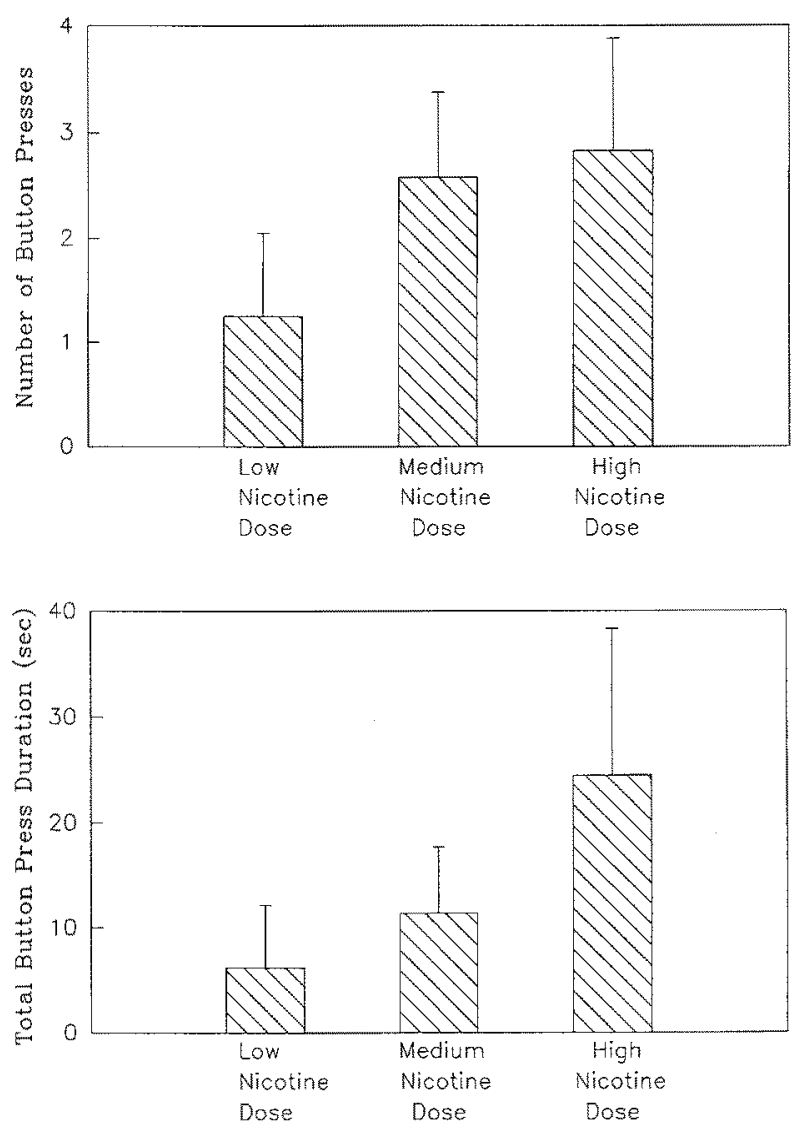

Fig. 3. Upper panel: total number of reports of euphoric sensations (button-presses) associated with low-, medium-, and high-nicotine dosing conditions via research cigarette following overnight deprivation (mean $+\operatorname{SEM}, N=12$ ). Lower panel: total duration of reports of euphoric sensations (time during which button was depressed) associated with low-, medium-, and high-nicotine dosing conditions via research cigarette following overnight deprivation (mean $\pm \mathrm{SEM}, N=12$ )

presses; total duration $6.2 \pm 6.0 \mathrm{~s}$ ) and higher but undifferentiated for the medium and high-nicotine cigarettes $(2.8 \pm 1.0$ and $2.6 \pm 0.8$ button-presses, respectively; $17.1 \pm 10.4$ and $18.7 \pm 11.5 \mathrm{~s}$ total duration, respectively).

Since all subjects received their lowest dose on the ultralow-nicotine day, we rearranged our medium and high conditions to reflect actual nicotine intake by switching the high and medium days for the six subjects who took in more nicotine from the medium- than from the high-nicotine cigarette. This manipulation produced a clean separation of the medium- and high-nicotine conditions, with plasma nicotine increments of $13.4 \pm$ $1.5 \mathrm{ng} / \mathrm{ml}$ and $22.8 \pm 3.8 \mathrm{ng} / \mathrm{ml}$, respectively. A highly significant linear trend was detected for these means $[F(1,11)=29.07, P<0.0005]$.

In the ultralow-nicotine dosing condition, only 4 of the 12 subjects ( $33 \%$ ) experienced any euphoric sensations. Corresponding figures for the medium-nicotine and high-nicotine dosing conditions were 10 subjects $(83 \%)$ and 11 subjects $(92 \%)$, respectively.

Figure 3 (upper panel) shows mean number of reports of euphoric sensations for each nicotine dose. A signifi- 
cant effect was observed for cigarette condition $[F(2,22)=5.87, P<0.01]$. Post hoc tests showed that number of reports of euphoric sensations presses for both the medium and high doses differed significantly from the ultra-low dose (low versus medium: $P<0.05$; low versus high: $P<0.05$ ). Although number of euphoric sensations for the medium and high doses did not differ significantly from each other, a significant linear trend emerged $[F(1,11)=8.50, P<0.05)$. For total duration of reports of euphoric sensations (Fig. 3, lower panel), a linear dose-response effect failed to reach significance.

\section{Combined data for first cigarette/high-nicotine condition}

In the combined sample of 22 subjects, the number of button-presses made when a high-nicotine cigarette was smoked as the first cigarette of the day was significantly correlated with age $(r=+0.47, P<0.05)$ and with number of years smoked $(r=+0.48, P<0.05)$. Total duration of button-presses was significantly correlated with total puff number $(r=+0.43, P<0.05)$ and, in the 12 subjects for whom cotinine levels were assayed, with cotinine $(r=+0.68, P<0.05)$. No significant correlation was observed between either number or total duration of button-presses and FTQ scores.

Nineteen of the 22 subjects experienced at least one euphoric sensation while smoking that cigarette. For those subjects, the mean ( $\pm S D$ ) number of buttonpresses was $2.8 \pm 2.5$, each button-press having a mean duration of $11.1 \pm 25.5 \mathrm{~s}$; for the entire cigarette, mean total time that the button was depressed was $35.1 \pm$ $77.7 \mathrm{~s}$. Puff data are missing for one of these subjects; for the remaining 18, the time from the first puff to first button-press was $142.5 \pm 95.6 \mathrm{~s}$. The first buttonpress did not occur until subjects had taken a mean of $7.6 \pm 3.6$ puffs. The duration of the puff that most recently preceded the first button-press was $4.8 \pm 9.6 \mathrm{~s}$, and the time that elapsed between puff and button-press was $25.1 \pm 36.6 \mathrm{~s}$.

\section{Discussion}

Our findings for both studies support and extend demonstrations previously reported in the literature that nicotine produces euphoric sensations in a dose-related manner. Although subjects failed to cooperate with our dosing manipulation (see Pomerleau et al. 1989) and managed, on average, to extract as much nicotine from their usual-brand cigarette and the medium-nicotine research cigarette as from the high-nicotine research cigarette (as evidenced by heart rate increases in experiment 1 and plasma nicotine boost in experiment 2), they were unable to obtain their presumably preferred nicotine dose from the ultralow-nicotine research cigarette and reported correspondingly less euphoria. Furthermore, when the data from experiment 2 are rearranged on the basis of nicotine plasma levels, an orderly doseresponse curve emerges for number of euphoric sensations. (It should be noted that puff-by-puff measures of plasma nicotine, or ideally, of brain and arterial levels, would help to substantiate this inference.)

Because the evidence regarding differential nicotine exposure for the usual-brand and high-nicotine cigarettes is ambiguous in experiment 1 , the additional impact of "familiarity" on the euphoriant effects of smoking cannot be determined. Clearly, however, euphoric sensations can be readily produced by unfamiliar research cigarettes, provided that they contain nicotine.

It should be noted that despite our use in our instructions of words like "high", "buzz", and "rush", which are widely used slang terms explicitedly associated with drug-induced euphoria, we cannot be certain that the "pleasurable effects" reproted by the subjects indeed constituted "euphoria." In future studies, it would probably be useful to include a post-study debriefing or some other subjective measures to provide a point of comparison and to ensure that subjects clearly distinguished these pleasurable sensations from feelings of dizziness, intoxication, or relaxation.

The results of experiment 1 are consistent with the possibility that tolerance to the euphoria-inducing properties of nicotine develops to subsequent dosing (along with tolerance to its cardiovascular effects), since the response to the second cigarette was considerably attenuated as compared to the response to the first cigarette. On the other hand, it is also possible that subjects confused "euphoria" with relief of withdrawal. In the absence of measures of plasma nicotine, moreover, we cannot rule out the possibility that the observed differences in heart rate or reports of euphoric sensations are due simply to self-administration of lower doses of nicotine during the second cigarette of the day, rather than to differences in either tolerance or withdrawal symptomatology.

Correlations between euphoria variables and smoking-related or demographic variables suggest that more experienced and more dependent smokers (as indicated by age, years smoked, and cotinine level, though not FTQ score) are more likely to experience euphoric sensations during smoking - possibly because these smokers self-administer more nicotine (Pomerleau et al. 1983), or because they have "learned" to self-administer it in patterns conducive to the production of euphoric sensations. A more systematic exploration of the relationship between nicotine dependence and the ability of nicotine to induce euphoria, in a larger number of subjects stratified for degree of dependence, would be necessary to substantiate this speculation.

The mean number of reports of euphoric sensations per high-nicotine cigarette (for those subjects who reported any such sensations) was around three per cigarette, each lasting a mean of around $11 \mathrm{~s}$. Thus, we can infer that these subjects experienced euphoric sensations for around $10 \%$ of the time involved in smoking a cigarette. Despite the fact that nicotine inhaled into the lungs reaches the brain in from 10 to $20 \mathrm{~s}$ (Benowitz 1988), the mean elapsed interval between onset of the first puff and onset of the first button-press was nearly $2.5 \mathrm{~min}$ - an interval comparable to, though slightly shorter than, that reported by Henningfield $(1983,1984)$. (The 
shortest interval for any subject was $29 \mathrm{~s}$, and the longest was $414 \mathrm{~s}$, well after the cigarette was finished.) Since the mean duration of the puff immediately preceding the first button-press was less than $5 \mathrm{~s}$, whereas the mean interval between onset of that puff and onset of the first button-press was nearly $30 \mathrm{~s}$, we conclude that the onset of a euphoric sensation typically does not occur during a puff. In view of the delay between puff and sensation, it may be that the observed euphoriant effects of nicotine result from the cascade of neuroregulatory and neuromodulatory effects initiated by the arrival in brain of a series of nicotine boli (see Pomerleau and Pomerleau 1984).

Our study suggests that because euphoric sensations are experienced for only $10 \%$ of the time that elapses during the smoking of a cigarette, the phenomenon must be studied under conditions of subject-initiated reporting; queries made only at specified, standardized intervals (as is typically done) may fail altogether to capture this effect - possibly explaining why this aspect of the smoking experience has been so largely neglected. Although controlled dosing methods undoubtedly help to regularize the temporal characteristics of this response, the extent to which variability can be reduced remains an empirical question; thus, both self-administration and parametric fixed dosing studies will be needed. Although experimental control of dosing has obvious advantages, it may be that smokers' ability to adjust dose to maximize the favorable effects of nicotine (and minimize the toxic effects), and to achieve them reliably on demand, are a critical component in the reinforcement for smoking behavior - perhaps even more important than the actual magnitude of the effects. Studies involving concomitant EEG measurement and studies using receptor antagonism (e.g., adrenergic or opiate blockers as well as mecamylamine) may also shed further light upon the ephemeral euphoriant effects of nicotine from smoking.

Acknowledgements. Support for this research was provided by $\mathrm{Na}-$ tional Cancer Institute grant CA-42730 and National Institute on Drug Abuse grant DA-06529 to the second author. The authors gratefully acknowledge the assistance of Susan M. Basson, Karen A. Flessland, and Deborah D. Kloska in carrying out this project.

\section{References}

Benowitz N (1988) Pharmacologic aspects of cigarette smoking and nicotine addiction NEJM 319:1318-1330
Dougherty JH, Miller D, Todd G, Kostenbauder HB (1981) Reinforcing and other behavioral effects of nicotine. Neurosci Biobehav Rev 5:487-495

Fagerstrom KO (1978) Measuring degree of physical dependence to tobacco smoking with reference to individualization of treatment. Addict Behav 3:235-241

Hariharan M, Van Noord T, Greden JF (1988) A high-performance liquid-chromatographic method for routine simultaneous determination of nicotine and cotinine in plasma. Clin Chem 34:724-729

Henningfield JE (1983) Measurement issues in cigarette smoking research: basic behavioral and physiological effects and patterns of nicotine self-administration. NIDA Res Monogr $48: 27-38$

Henningfield JE (1984) Pharmacologic basis and treatment of cigarette smoking. J Clin Psychiatry 45:12 [sec. 2]:24-34

Henningfield JE, Goldberg SR, Herning RI, Jasinski DR, Lukas SE, Miyasato K et al. (1986) Human studies of the behavioral pharmacological determinants of nicotine dependence. Problems of drug dependence 1985. NIDA Research Monograph 67, DHHS Publication (ADM) 86-1448. US Government Printing Office, Washington $D C$

Henningfield JE, Jasinski DR (1983) Human pharmacology of nicotine. Psychopharmacol Bull 19:413-415

Henningfield JE, London ED, Jaffe JH (1987) Nicotine reward: studies of abuse liability and physical dependence potential. In: Engel J, Oreland L (eds) Brain reward systems and abuse. Raven Press, New York.

Hubbard JE, Gohd RS (1975) Tolerance development to the arousal effects of nicotine. Pharmacol Biochem Behav 3:471-476

Jasinski DR, Boren JJ, Henningfield JE, Johnson RE, Lange WR, Lukas SE (1984) Progress report from the NIDA Addiction Research Center, Baltimore, Maryland. NIDA Res Monogr $49: 69-76$

Kozlowski LT, Wilkinson A, Skinner W, Kent C, Franklin T, Pope M (1989) Comparing tobacco cigarette dependence with other drug dependencies. JAMA $261: 898-901$

Lukas SE, Mendelson JH, Benedikt RA, Jones B (1986) EEG, physiologic and behavioral effects of ethanol administration. Problems of Drug Dependence 1985. NIDA Research Monograph 67, DHHS Publication (ADM) 86-1448. US Government Printing Office, Washington DC

Pickworth WB, Herning RI, Henningfield JE (1988) Mecamylamine reduces some EEG effects of nicotine chewing gum in humans. Pharmacol Biochem Behav 30:149-153

Pomerleau OF, Fertig J, Shanahan SO (1983) Nicotine dependence in cigarette smoking:an empirically-based, multivariate model. Pharmacol Biochem Behav 19:291-299

Pomerleau OF, Pomerleau CS (1984) Neuroregulators and the reinforcement of smoking: towards a biobehavioral explanation. Neurosci Biobehav Rev 8:503-513

Pomerleau OF, Pomerleau CS, Rose JE (1989) Controlled dosing of nicotine: a review of problems and progress. Ann Behav Med 11:158-163

US Department of Health and Human Services (USDHHS : 1988) The health consequences of smoking: nicotine addiction. A report of the Surgeon General. DHHS 88-8406. US Government Printing Office, Washington, DC 